Journal for ImmunoTherapy of Cancer

\section{Ipilimumab and nivolumab/ pembrolizumab in advanced hepatocellular carcinoma refractory to prior immune checkpoint inhibitors}

To cite: Wong JSL, Kwok GGW, Tang V, et al. Ipilimumab and nivolumab/pembrolizumab in advanced hepatocellular carcinoma refractory to prior immune checkpoint inhibitors. Journal for ImmunoTherapy of Cancer 2021;9:e001945. doi:10.1136/jitc-2020-001945

- Additional material is published online only. To view please visit the journal online (http://dx.doi.org/10.1136/jitc2020-001945).

The American Society of Clinical Oncology 2021 Gastrointestinal Cancer Symposium.

Accepted 26 December 2020

Check for updates

(C) Author(s) (or their employer(s)) 2021. Re-use permitted under CC BY-NC. No commercial re-use. See rights and permissions. Published by BMJ.

${ }^{1}$ Department of Medicine, Queen Mary Hospital, The University of Hong Kong, Hong Kong, China

${ }^{2}$ Department of Surgery, Queen Mary Hospital, The University of Hong Kong, Hong Kong, China

Correspondence to

Dr Thomas Yau; tyaucc@hku.hk

\section{ABSTRACT}

Background Programmed cell death protein 1 (PD-1) pathway blockade with immune checkpoint inhibitors (ICls) is a standard therapy in advanced hepatocellular carcinoma (HCC) nowadays. No strategies to overcome $\mathrm{ICI}$ resistance have been described. We aimed to evaluate the use of ipilimumab and anti-PD-1 ICls (nivolumab or pembrolizumab) combinations in patients with advanced HCC with progression on prior ICls.

Methods Patients with advanced HCC with documented tumor progression on prior ICls and subsequently received ipilimumab with nivolumab/pembrolizumab were analyzed. Objective response rate (ORR), median duration of response (DOR), time-to-progression (TTP), overall survival (OS), and treatment-related adverse events (TRAEs) were assessed.

Results Twenty-five patients were included. The median age was 62 (range: 51-83). About $68 \%$ were of Child-Pugh (CP) Grade A and 48\% had primary resistance to prior ICI. At median follow-up of 37.7 months, the ORR was $16 \%$ with a median DOR of 11.5 months (range: 2.76-30.3). Three patients achieved complete response. The median TTP was 2.96 months (95\% Cl: 1.61 to 4.31$)$. Median OS was 10.9 months (95\% Cl: 3.99 to 17.8$)$ and the 1 year, 2 year and 3 year survival rates were $42.4 \%, 32.3 \%$ and $21.6 \%$, respectively. The ORR was $16.7 \%$ in primary resistance group and $15.4 \%$ in acquired resistance group $(p=1.00)$. All responders were of CP A and AlbuminBilirubin (ALBI) Grade 1 or 2. CP and ALBI Grades were significantly associated with $0 S(p=0.006$ and $p<0.001$, respectively). Overall, $52 \%$ of patients experienced TRAEs and $12 \%$ experienced Grade 3 or above TRAEs.

Conclusions Ipilimumab and nivolumab/pembrolizumab can achieve durable antitumor activity and encouraging survival outcomes with acceptable toxicity in patients with advanced HCC who had prior treatment with ICls.

\section{BACKGROUND}

Liver cancer is the fourth leading cause of cancer death worldwide, with the majority (75\%-85\%) being hepatocellular carcinoma (HCC). ${ }^{1}$ HCC often arises from a microenvironment characterized by chronic inflammation, intrinsic immunosuppression and overexpression of immune checkpoints including the programmed cell death protein 1 (PD-1) pathway and programmed death ligand 1 (PDL-1), and the cytotoxic T-lymphocyte-associated protein 4 (CTLA-4) pathway. This results in reduction of proliferation, inhibition of activation and functional exhaustion of $\mathrm{T}$ cells leading to tumor immune escape. Targeting these characteristics, immune checkpoint inhibitors (ICIs) have significantly advanced the systemic treatment of inoperable, advanced HCC. Nivolumab, an anti-PD-1 antibody, demonstrated good efficacy and safety as secondline treatment for advanced HCC in the CheckMate-040 trial. ${ }^{2}$ Nivolumab as first-line treatment for advanced HCC also demonstrated clinical benefits when compared with sorafenib in the CheckMate-459 trial, although statistical significance was not obtained. $^{3} \quad$ In addition, pembrolizumab received accelerated regulatory approval for second-line treatment of advanced HCC based on the Keynote-224 trial. ${ }^{4}$ Nevertheless, in the subsequent Keynote-240 trial, both the median progression-free survival (PFS) and overall survival (OS) of pembrolizumab did not reach statistical significance per specified criteria. ${ }^{5}$ Recently, atezolizumab (an anti-PDL1-antibody) in combination with bevacizumab was approved for first-line treatment of advanced HCC based on results from the IMbrave150 trial, which demonstrated an impressive objective response rate (ORR) of $27.3 \%$, median PFS of 6.8 months and OS at 12 months of $67.2 \%$, all superior to the sorafenib arm. ${ }^{6}$ Based on these emerging data, ICIs will be increasingly used in both first-line and second-line settings for the treatment of patients with advanced HCC. Importantly, the majority of patients treated 
Table 1 Baseline characteristics

\section{Patient characteristics}

\begin{tabular}{|c|c|}
\hline Median age (range), years & $62(51-83)$ \\
\hline Male, n (\%) & $22(88 \%)$ \\
\hline \multicolumn{2}{|l|}{ HCC etiology, n (\%) } \\
\hline Hepatitis B (HBV) & $19(76 \%)$ \\
\hline Hepatitis C (HCV) & $2(8 \%)$ \\
\hline Alcoholic & $1(4 \%)$ \\
\hline NASH & $2(8 \%)$ \\
\hline Cryptogenic & $1(4 \%)$ \\
\hline \multicolumn{2}{|l|}{ BCLC stage, n (\%) } \\
\hline $\mathrm{B}$ & $4(16 \%)$ \\
\hline $\mathrm{C}$ & $19(76 \%)$ \\
\hline $\mathrm{D}$ & $2(8 \%)$ \\
\hline Extrahepatic metastases, n (\%) & $20(80 \%)$ \\
\hline Vascular invasion, n (\%) & $3(12 \%)$ \\
\hline AFP $\geq 400 \mu \mathrm{g} / \mathrm{L}, \mathrm{n}(\%)$ & $11(44 \%)$ \\
\hline
\end{tabular}

CP Grade

$\begin{array}{cc}\text { A } & 17(68 \%) \\ \text { B } & 6(24 \%) \\ \text { C } & 2(8 \%)\end{array}$

\section{ALBI Grade}

$\begin{array}{rr}1 & 9(36 \%) \\ 2 & 11(44 \%) \\ 3 & 5(20 \%)\end{array}$

Baseline performance status

\begin{tabular}{|cc}
\hline $0-1$ & $23(92 \%)$ \\
\hline 2 & $2(8 \%)$ \\
\hline Prior ICI & $19(76 \%)$ \\
\hline Nivolumab & $5(20 \%)$ \\
\hline Pembrolizumab & $1(4 \%)$ \\
\hline Atezolizumab with bevacizumab & \\
\hline Agents in combination with prior ICI & $21(84 \%)$ \\
\hline None & $2(8 \%)$ \\
\hline Bevacizumab & $1(4 \%)$ \\
\hline Capecitabine, oxaliplatin, irinotecan & $1(4 \%)$ \\
\hline Cabozantinib & $12(48 \%)$ \\
\hline Primary resistance to prior ICI & \\
\hline Lines of systemic therapies prior to & $15(60 \%)$ \\
\hline ipilimumab + anti-PD-1, $\mathrm{n}(\%)$ & $7(28 \%)$ \\
\hline 1 & $3(12 \%)$ \\
\hline 2 & $19(76 \%)$ \\
\hline 3 & \\
\hline Therapies prior to any ICI, $\mathrm{n}(\%)$ & \\
\hline Prior local treatment & \\
\hline Curative surgical resection & \\
\hline
\end{tabular}

Continued
Table 1 Continued

\begin{tabular}{lc}
\hline Patient characteristics & \\
\hline TACE & $15(60 \%)$ \\
\hline Prior systemic treatment & $10(40 \%)$ \\
Sorafenib & $7(28 \%)$ \\
Lenvatinib & $1(4 \%)$ \\
Enzalutamide & $1(4 \%)$ \\
PEG-BCT-100, oxaliplatin, capecitabine & $1(4 \%)$ \\
FGF401 & $1(4 \%)$ \\
\hline
\end{tabular}

AFP, alpha-fetoprotein; ALBI, Albumin-Bilirubin; BCLC, Barcelona clinic liver cancer; $\mathrm{CP}$, Child-Pugh; HCC, hepatocellular carcinoma; $\mathrm{ICl}$, immune checkpoint inhibitor; NASH, non-alcoholic steatohepatitis; PD-1, programmed cell death protein 1; TACE, Transarterial chemoembolization.

with ICIs will either never respond (primary resistance) or eventually develop resistance (acquired resistance). Hence, there is an unmet need to investigate the appropriate treatment for the rapidly expanding group of patients with advanced HCC who had tumor progression on prior anti-PD-1/L1 therapies.

To improve the therapeutic efficacy of ICIs, anti-CTLA-4 and anti-PD-1/L-1 are increasingly used simultaneously, with the combination of ipilimumab (an anti-CTLA-4 antibody) and nivolumab having received Food and Drug Administration approval in various cancer types. In patients with advanced HCC previously treated with sorafenib, ipilimumab-nivolumab has also received regulatory approval based on the promising results of the CheckMate-040 cohort 4 trial which reported an ORR of up to $32 \%$ and a median OS of up to 23 months. ${ }^{7}$ Recently, durvalumab (an anti-PDL-1 antibody) in combination with tremelimumab (an anti-CTLA-4 antibody) reported an ORR of up to $22.7 \%$ and a median OS of up to 18.7 months in population with advanced HCC. ${ }^{8}$ Interestingly, in advanced melanoma, non-small-cell lung cancer and metastatic renal cell carcinoma, salvage ipilimumab and nivolumab have lately demonstrated promising anti-tumor activities in patients with prior PD-1 pathway blockade. ${ }^{9-12}$

To evaluate the efficacy and safety of combined CTLA-4 and PD-1 blockade in patients with advanced HCC who failed PD-1 pathway blockade, we conducted an analysis of patients who received a combination of ipilimumab and nivolumab/pembrolizumab after tumor progression on prior anti-PD-1/L-1.

\section{METHODS}

This was a single-center retrospective analysis approved by The University of Hong Kong/Hospital Authority Hong Kong West Cluster Institutional Review Board. Patients were identified from a prospectively maintained HCC database at Queen Mary Hospital, Hong Kong. Between June 2016 and February 2020, consecutive patients with 
HCC who were ineligible for surgical or locoregional therapies and had documented tumor progression on anti-PD-1/L-1 were included for analysis. All included patients received at least one dose of a combination of ipilimumab and nivolumab/pembrolizumab. Patients who received any other concomitant anticancer therapy were excluded.

HCCs were diagnosed either by histological confirmation, or according to the European Association for the Study of the Liver combined criteria if both characteristic radiological findings and an elevated serum alphafetoprotein level were present. Fine-needle cytology or biopsy was used for histological confirmation in cases of diagnostic uncertainty. Tumors were staged according to the Barcelona Clinic Liver Cancer system by CT scan or by MRI. Clinical, radiological and laboratory information were gathered from the database.

\section{Treatment schedule, disease evaluation and follow-up}

All patients received ipilimumab $1 \mathrm{mg} / \mathrm{kg}$ with nivolumab $3 \mathrm{mg} / \mathrm{kg}$ or pembrolizumab $2 \mathrm{mg} / \mathrm{kg}$ scheduled every 3 weeks. In general, four induction doses of ipilimumab and nivolumab/pembrolizumab followed by 1 year of maintenance nivolumab/pembrolizumab were scheduled. In selected cases, ipilimumab and nivolumab/pembrolizumab was administrated beyond four cycles at the discretion of treating clinicians. Radiological investigations were evaluated by investigators according to the Response evaluation criteria in solid tumors V.1.1 (RECIST 1.1) and the modified RECIST 1.1 for immune-based therapeutics (iRECIST).${ }^{13}$ Best objective response (BOR) was defined as the best response per RECIST V.1.1 compared with baseline. ORR was defined as the percentage of patients with complete response (CR) or partial response (PR) according to RECIST 1.1. Patients who achieved CR, PR or stable disease (SD) were defined as achieving clinical benefits. Radiological reassessments of tumor response were scheduled every 8 to 12 weeks after treatment initiation. Primary resistance was defined as always having progressive disease on prior ICI, while acquired resistance was defined as ever having radiological CR, PR or SD with prior ICI. Treatment-related adverse events (TRAEs) were graded using the National Cancer Institute Common Terminology Criteria for Adverse Events (V.4.0). ${ }^{15}$ All patients' records were reviewed up to the end of their follow-up periods or study cut-off to observe for delayed TRAEs. TRAE-related ICI discontinuation or delay and systemic immunosuppressive treatments were recorded. All patients were followed up with regular clinical examinations, blood tests and scanning until death or last follow-up.

\section{Statistical analysis}

All statistical analyses were performed using SPSS (V.26, IBM, New York, USA). Duration of response (DOR) was calculated from the earliest date of CR or PR to progressive disease or last follow-up if no progression. Follow-up time was calculated from the date of first dose to death or last clinic follow-up. Time-to-progression (TTP) was calculated from the date of first dose to radiological progression, or censored at date of death or last follow-up while on treatment for patients without progression. OS was calculated from the date of first dose to death of any cause or censored at last follow-up for patients who were still alive at study cut-off. Median TTP, median OS and survival rates were estimated by Kaplan-Meier analyses and survival curves were compared using log-rank tests. Median follow-up was estimated by Reverse Kaplan-Meier analysis using follow-up time. Categorical variables were compared using Pearson's $\chi^{2}$ test or Fisher's exact test. A $\mathrm{p}$ value of 0.05 was used as the cut-off for statistical significance.

\section{RESULTS \\ Demographics}

Twenty-five patients were included in the analysis. Table 1 shows their baseline characteristics. The median age was 62 (range 51-83). The majority of patients were of ChildPugh (CP) Grade A (68\%) and 36\% were of AlbuminBilirubin (ALBI) Grade 1. About 76\% had Hepatitis B-related HCC. Regarding prior ICIs, 96\% used anti-PD-1 antibodies (nivolumab 76\%, pembrolizumab 20\%) while $4 \%$ used atezolizumab with bevacizumab. The majority $(84 \%)$ of patients received prior ICI as mono therapy. The median time on prior ICI was 4.86 months (range $0-21.2$ ) and $48 \%$ had primary resistance. Most patients had documented radiological progression on prior ICI. Overall, $48 \%$ received ipilimumab with nivolumab and $52 \%$ received ipilimumab with pembrolizumab. Online supplemental table 1 shows the combined ICI regime according to prior ICI treatment. Of note, $60 \%$ of patients received ipilimumab and nivolumab/pembrolizumab as second-line, $28 \%$ as third-line and $12 \%$ as fourth-line systemic treatment. The median interval between the last dose of prior ICI and the first dose of ipilimumab and nivolumab/pembrolizumab was 3.00 weeks (range $1.29-13.0)$.

\section{Clinical outcomes}

The median follow-up was 37.7 months (95\% CI: 32.8 to 42.7). At the study cut-off date, $28 \%$ of patients were still alive. No patients were lost to follow-up. Table 2 summarizes BORs. Overall, ORR was $16 \%$, with three $(12 \%)$ patients having CR and one (4\%) patient having PR as their BORs. Another six (24\%) patients achieved SD. Thus, $40 \%$ of patients achieved clinical benefits. The median DOR was 11.5 months (range 2.76-30.3). Two patients of DOR 10.6 months and 30.3 months continued to be in CR at the study cut-off date. One patient with CR had treatment suspension after two cycles due to TRAE and had tumor recurrence after a DOR of 12.4 months. There were no differences in the BOR of any patients using the RECIST 1.1 and iRECIST criteria.

The median TTP was 2.96 months (95\% CI: 1.61 to 4.31). Overall, the median OS was 10.9 months (95\% 


\begin{tabular}{lc|}
\hline Table 2 & Best objective response \\
\hline Activity & $\mathbf{n}(\%)$ \\
\hline CR & $3(12)$ \\
PR & $1(4)$ \\
SD & $6(24)$ \\
PD & $12(48)$ \\
Non-evaluable & $3(12)$ \\
ORR (\%) & $4(16)$ \\
\hline
\end{tabular}

$\mathrm{CR}$, complete response; ORR, objective response rate; $\mathrm{PD}$, progressive disease; PR, partial response; SD, stable disease.

CI: 3.99 to 17.8 , figure 1). The 1-year, 2-year and 3-year survival rates were $42.4 \%, 32.3 \%$ and $21.6 \%$, respectively. $40 \%$ of patients received other subsequent systemic therapies.

The ORR of patients with primary resistance to prior ICI was $16.7 \%$ versus $15.4 \%$ of those with prior acquired resistance $(p=1.00)$. Nevertheless, patients with prior primary resistance had a median OS of 4.44 months (95\% CI: 0.00 to 13.2$)$, compared with 11.4 months $(95 \%$ CI: 3.67 to 19.1$)$ of those with prior acquired resistance $(p=0.55$, figure 2$)$. Importantly, all responders were of CP $\mathrm{A}$ at first dose. About $50 \%$ of responders were of ALBI Grade 1 and the rest Grade 2. Baseline CP Grade (A vs $\mathrm{B}$ or $\mathrm{C}$ ) was significantly associated with OS (median OS 16.2 months (95\% CI: 7.56 to 24.8$)$ vs 1.87 months (95\% CI: 1.28 to 2.47 ), $\mathrm{p}=0.006$, figure 3 ). Baseline ALBI Grade was also significantly associated with OS (median OS Grade 1 not reached, Grade 210.9 months (95\% CI: 5.91 to 15.9 ), Grade 31.68 months (95\% CI: 0.00 to 4.14 ), $\mathrm{p}<0.001$, figure 4 ).

\section{Dosing, safety and TRAEs}

The median dose received was three (range 1-22). Only $36 \%$ of patients completed four doses of induction ipilimumab and nivolumab/pembrolizumab. No delay in dosing was observed. About $48 \%$ of patients stopped treatment due to progressive disease, $16 \%$ due to TRAEs, $16 \%$ due to non-cancer death and $8 \%$ due to infections and other unrelated causes. About $8 \%$ of patients were still receiving therapy at study cut-off.

Table 3 shows all TRAEs that occurred in the analysis. Overall, $52 \%$ of patients experienced TRAEs. The most prevalent TRAE was skin-related (32\%) followed by endocrinopathies (20\%). Regarding Grade 3-4 TRAEs, one patient had Grade 3 hepatitis, and another had Grade 4 colitis. In total, three (12\%) patients required systemic immunosuppressants. All three received corticosteroids. The median duration of corticosteroids given was 100 days (range 7-185) and the median initiation dose was $30 \mathrm{mg}$ prednisolone (range 5-37.5). In addition, the patient with Grade 4 colitis received concomitant budesonide and mycophenolate mofetil, while a patient with toxic epidermal necrolysis (TEN) received concomitant intravenous immunoglobulin therapy. The patients with Grade 3 hepatitis and Grade 4 colitis recovered after systemic immunosuppressants without any long-term sequelae. One Grade 5 fatal TRAE occurred in a patient who developed TEN after the first dose of ipilimumab and nivolumab. Any grade TRAE was significantly associated with OS (median OS any TRAE 16.2 months $(95 \%$ CI: 8.38 to 24.0 ) vs no TRAE 2.10 months (95\% CI: 0.93 to $3.27), \mathrm{p}=0.024$, online supplemental figure 1). However, there was no association between any TRAE and objective responses $(p=1.00)$ or clinical benefit $(p=0.69)$.

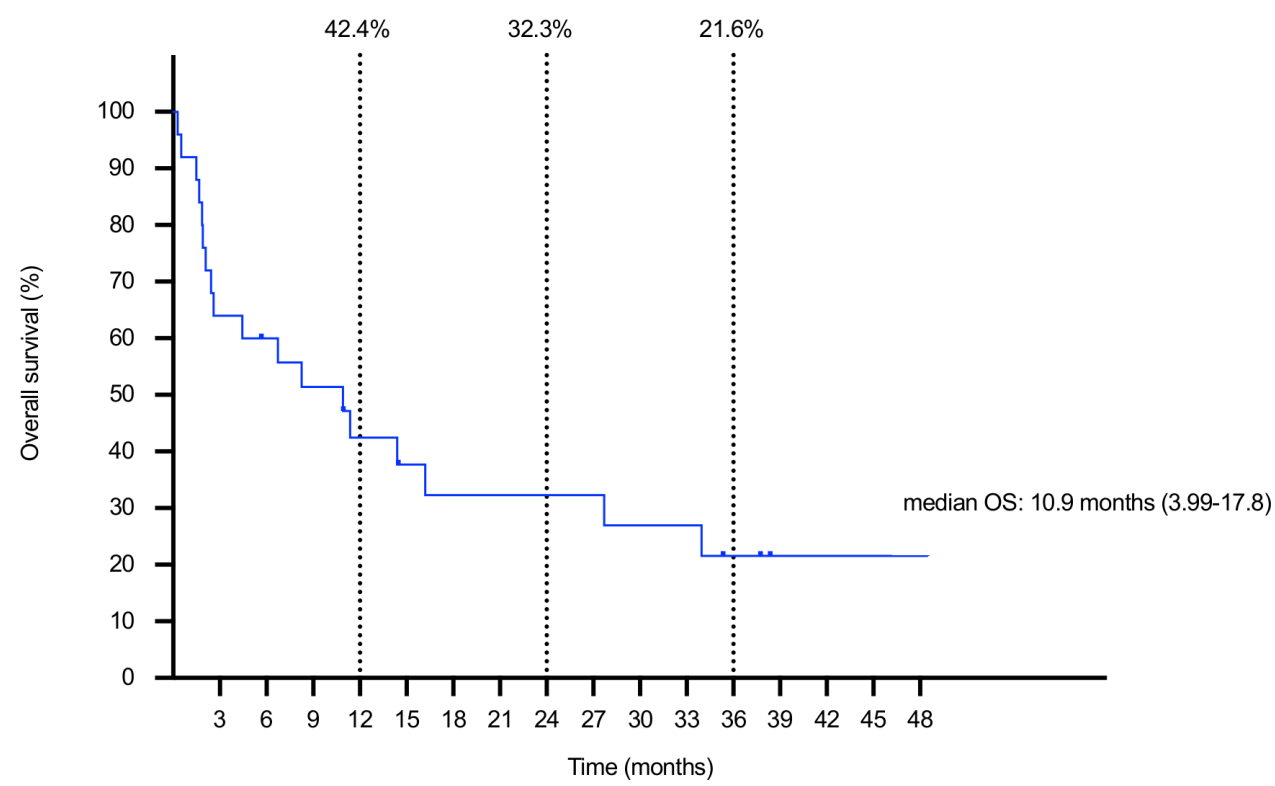

Figure 1 Kaplan-Meier analysis of OS. Dotted lines plot time points, percentages represent survival rates. OS, overall survival. 


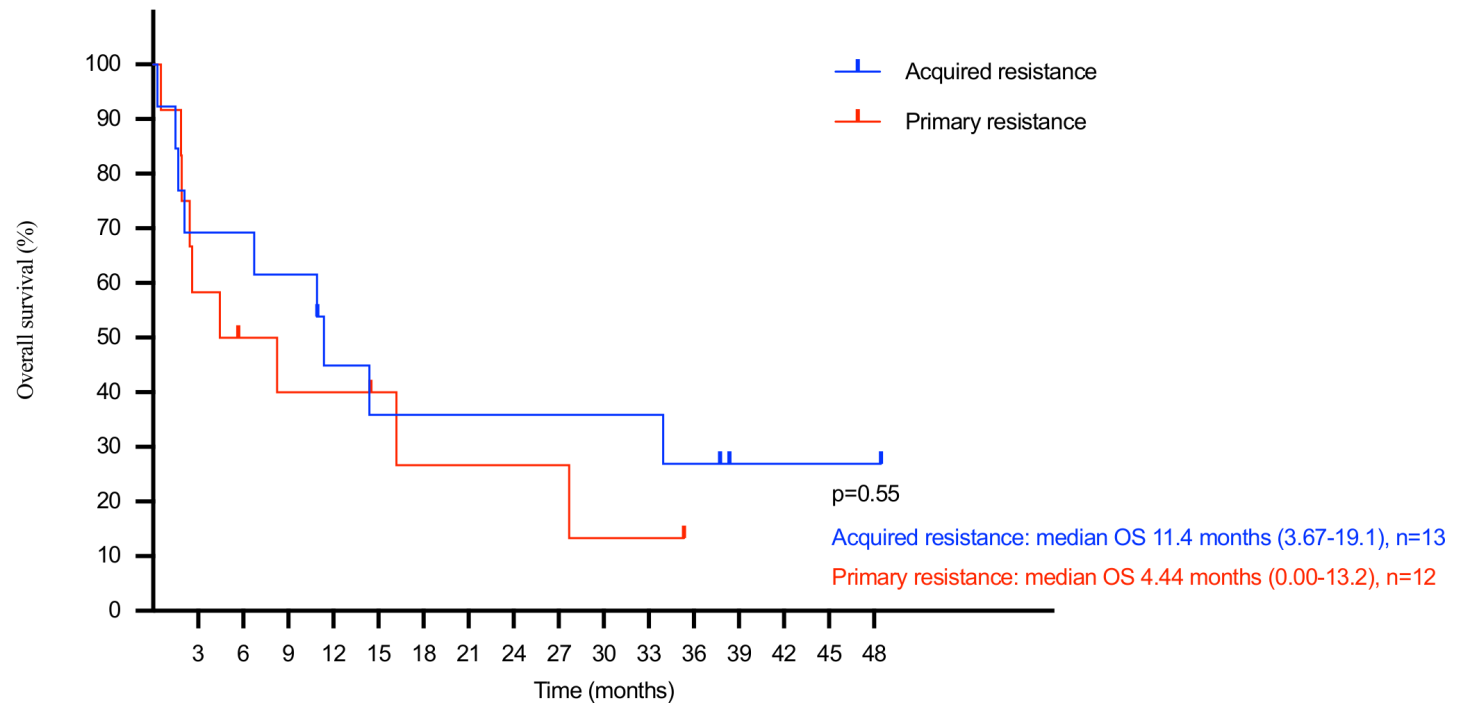

Figure 2 Kaplan-Meier analysis of OS by resistance pattern to prior ICI. Level of significance: p=0.55 (log-rank test). ICI, immune checkpoint inhibitor; OS, overall survival.

\section{DISCUSSION}

Our present analysis reports an overall ORR of $16 \%$ and median OS of 10.9 months after a long-term follow-up of 37.7 months in patients with advanced HCC treated with ipilimumab and nivolumab/pembrolizumab after tumor progression on prior anti-PD-1/L1. Importantly, three $(12 \%)$ patients achieved CR. Responses were also durable, with a median DOR of 11.5 months. These results are encouraging as the majority of analyzed patients were heavily pretreated with poor prognosis. More importantly, these reported ORR, DOR and OS provide important information to address the emergent clinical problem of how to treat patients with anti-PD-1/ L1 refractory advanced HCC.

In fact, salvage ipilimumab with nivolumab after prior anti-PD-1/L1 has been described in patients with renal cell carcinoma (RCC), melanoma and non-small cell lung cancer (NSCLC) ${ }^{9-12}$ All these studies demonstrated ORRs of about $10 \%-20 \%$ and similar TRAE incidences compared with our analysis. The observation that response rates to ipilimumab-anti-PD-1 were similar regardless of prior anti-PD-1/L1 response was also shared by two such studies. ${ }^{9}$ Notably, our analysis demonstrated an impressive $\mathrm{CR}$ rate $(12 \%)$. Multiple prospective clinical trials are currently underway to investigate ipilimumab-nivolumab after prior PD-1/L1 blockade for patients with RCC, ${ }^{16-18}$ NSCLC $^{1920}$ and melanoma. ${ }^{21}$ Nevertheless, no comparable prospective trial in patients with advanced HCC exists to the best of our knowledge.

Mechanistically, the effects of the PD-1 pathway on T cells occur predominantly during the effector phase. In contrast, the CTLA-4 pathway affects the immune priming and proliferation phases of $\mathrm{T}$ cells. ${ }^{22}$ Distinct genomic and functional signatures in $\mathrm{T}$ cells have been

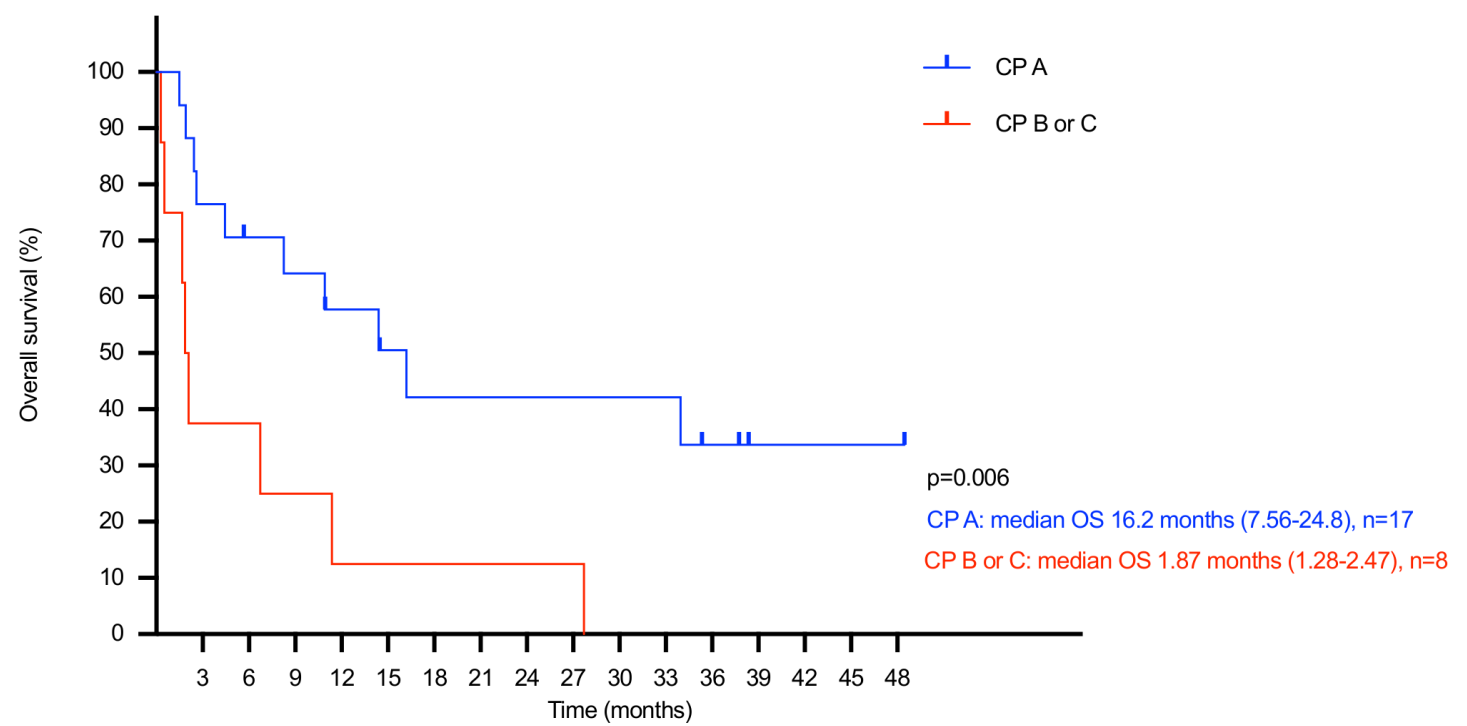

Figure 3 Kaplan-Meier analysis of OS by CP Grade. Level of significance: $\mathrm{p}=0.006$ (log-rank test). CP, Child-Pugh; OS, overall survival. 


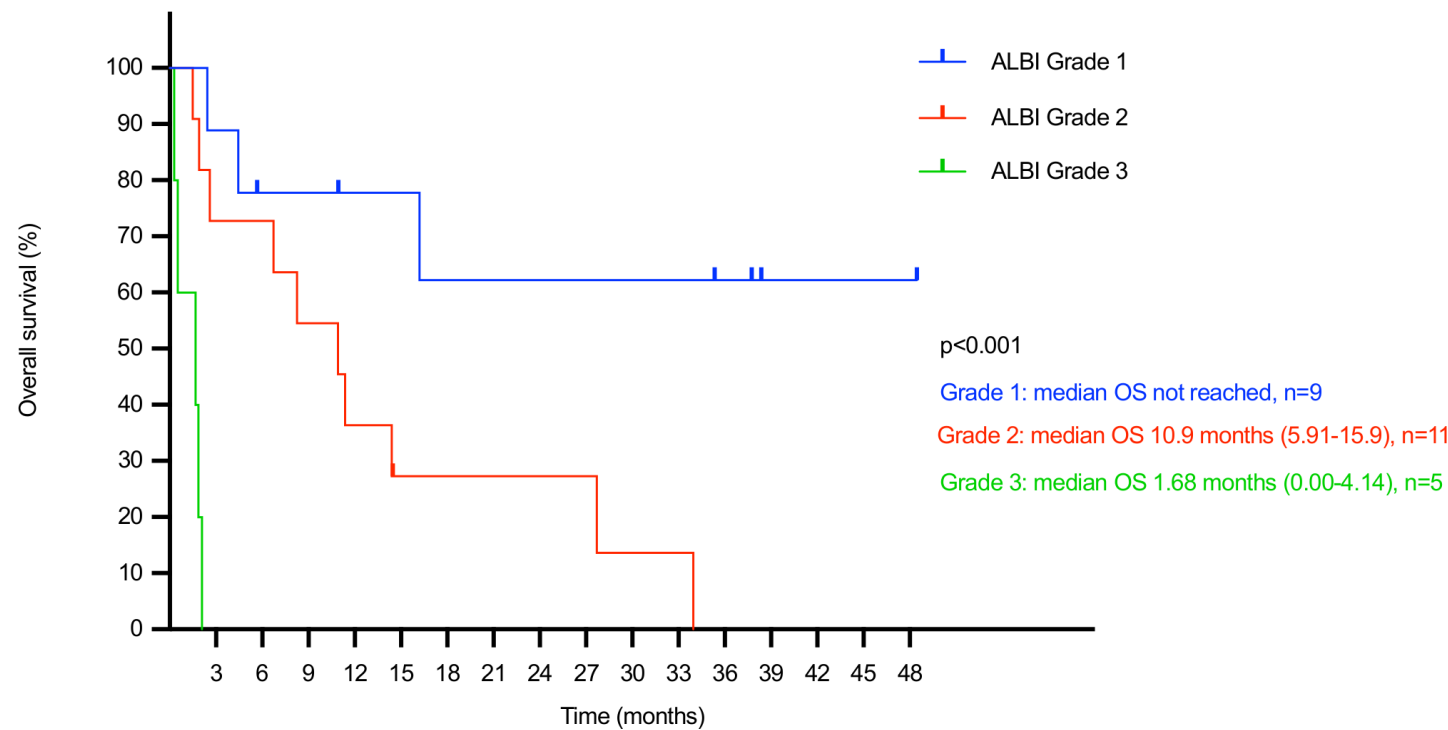

Figure 4 Kaplan-Meier analysis of OS by ALBI Grade. Level of significance: $p<0.001$ (log-rank test). ALBI, Albumin-Bilirubin; OS, overall survival.

observed in combination blockade compared with single PD-1 pathway blockade. ${ }^{23}$ The following mechanisms may provide the scientific rationale for the clinical benefits of salvage combined CTLA-4 and PD-1 blockade in patients resistant to single PD-1 pathway blockade. In patients with primary resistance to single PD-1 pathway blockade, an immunosuppressive tumor microenvironment due to the presence of myeloid-derived suppressor cells (MDSCs) and regulatory $\mathrm{T}$ cells (Treg) has been established as a key factor of tumor immune escape. ${ }^{24-26}$ Compared with single PD-1 pathway blockade, combined CTLA-4 and PD-1 blockade has been shown to increase the CD $8+\mathrm{T}$ cell to MDSC ratio, as well as significantly decrease the fraction of Tregs expressing activation markers 4-1BB and KLRG $1 .{ }^{27}$ On the other hand, in patients with acquired resistance to single PD-1 pathway blockade, PD-1 pathway blockade causes subsequent upregulation of alternative immune checkpoints such as CTLA- $4,{ }^{28}$ which may be reversed by CTLA- 4 blockade. Furthermore, PD-1 pathway blockade does not activate genes associated with memory $\mathrm{T}$ cells, which have a greater longevity and proliferative capacity than effector T cells and confer longerterm tumor rejection. ${ }^{23}{ }^{29}$ CTLA-4 blockade is associated with proliferation of transition memory $\mathrm{T}$ cells, ${ }^{23}$ which may provide more long-term tumor control. This may also explain the durability of response observed in this analysis, especially for the three patients in CR.

In this analysis, all responders were of $\mathrm{CP} A$ and of ALBI Grade 1 or 2. In addition, CP and ALBI grades were significantly associated with OS. Cirrhosis has been postulated to contribute to tumor immune escape through several mechanisms. First, cirrhotic livers have an increased extracellular matrix (ECM) content. ${ }^{30}$ ECM proteins may activate integrins causing the activation of transforming growth factor beta, a potent suppressor of anti-tumor immunity. ${ }^{31}$ ECM may also provide a physical barrier against immune cell infiltration. ${ }^{32}$ Second, liver fibrogenesis is mediated by activation of hepatic stellate cells (HSCs) ${ }^{33}$ HSCs have been shown to inhibit tumor lymphocytic infiltration, induce immunosuppressive cytokines, and induce the differentiation of both Treg and MDSCs, thus inhibiting both adaptive and innate immune responses. ${ }^{34}$ This analysis is in line with the above observations in its suggestion that the response to ICIs may be affected by the extent of cirrhosis. Interestingly, the CheckMate-040 cohort 5 study demonstrated that

Table 3 Treatment related adverse events

\begin{tabular}{lllll}
\hline & Grade 1-2 & Grade 3-4 & Grade 5 & Total \\
\hline Any TRAE, patients (\%) & & & & $13(52 \%)$ \\
Adverse events, $\mathrm{n}(\%)$ & & & & \\
$\quad$ Skin related & $7(28 \%)$ & 0 & $1(4 \%)$ & $8(32 \%)$ \\
Hepatitis & 0 & $1(4 \%)$ & 0 & $1(4 \%)$ \\
Colitis & $1(4 \%)$ & $1(4 \%)$ & 0 & $2(8 \%)$ \\
Hypothyroidism/adrenal insufficiency & $5(20 \%)$ & 0 & 0 & $5(20 \%)$ \\
Constitutional & $2(8 \%)$ & 0 & 0 & $2(8 \%)$ \\
\hline
\end{tabular}

TRAE, treatment-related adverse event. 
although patients with CP B advanced HCC might derive clinical benefits from single agent nivolumab, benefits were mostly limited to patients with CP B7 advanced HCC and survival benefits were less pronounced compared with patients with CP A advanced HCC..$^{35}$ Further studies are needed to better illustrate the relationship between the degree of cirrhosis and ICI response.

Interestingly, the ORRs to ipilimumab and nivolumab/ pembrolizumab in this analysis were similar regardless of whether the patient had primary or acquired resistance to prior anti-PD-1/L1. However, the median OS was much longer in patients with prior acquired resistance compared with those with primary resistance, although this was not statistically significant. In fact, the difference in OS might partly be due to differences in tumor biology: primary resistance patients may have more advanced tumor immune escape, resulting in quicker tumor progression and earlier death compared with acquired resistance patients. More importantly, our findings might imply that using ipilimumab and nivolumab/pembrolizumab might have better efficacy in patients with acquired resistance to prior anti-PD-1/L1 compared with primary resistance cases. The non-significant statistical result observed here was likely due to the small number of patients analyzed.

It is worthwhile to note that all patients in this analysis received ipilimumab at $1 \mathrm{mg} / \mathrm{kg}$. There is evidence that the dose of ipilimumab and anti-PD-1, when used in combination, affects the OS of patients with advanced HCC. An analysis of the CheckMate-040 trial cohort 4 showed that among patients with advanced HCC treated with ipilimumab and nivolumab after sorafenib, the ipilimumab $3 \mathrm{mg} / \mathrm{kg}$ and nivolumab $1 \mathrm{mg} / \mathrm{kg}$ every 3 weeks arm achieved a median OS of 22.8 months and $8 \%$ CR rate, compared with 12.5 months and $6 \%$ in the ipilimumab $1 \mathrm{mg} / \mathrm{kg}$ and nivolumab $3 \mathrm{mg} / \mathrm{kg}$ every 3 weeks arm, and 12.7 months and $0 \%$ in the nivolumab $3 \mathrm{mg} /$ $\mathrm{kg}$ every 2 weeks and ipilimumab $1 \mathrm{mg} / \mathrm{kg}$ every 6 weeks arm. ${ }^{7}$ In small cell lung cancer, improved OS was also reported with a higher ipilimumab dose. ${ }^{36}$ However, the important question of whether higher doses of ipilimumab (such as $3 \mathrm{mg} / \mathrm{kg}$ ) may achieve even better ORRs and survival outcomes in patients with anti-PD-1/L1 resistance remains. Further mechanistic studies and clinical trials are warranted.

Overall, ipilimumab and nivolumab/pembrolizumab were well-tolerated, with only three (12\%) patients having $\geq$ Grade 3 TRAEs. Nevertheless, one treatment-related death occurred. The pattern and severity of TRAEs reported in this analysis are comparable to those of ipilimumab $1 \mathrm{mg} / \mathrm{kg}$ arms in published trials and lower than those of ipilimumab $3 \mathrm{mg} / \mathrm{kg}$ arms, consistent with trial observations that higher ipilimumab dosages are associated with increased incidence and severity of TRAEs. ${ }^{37-41}$ Patients who experienced any TRAE were observed to have superior OS compared with those who did not. Several possible explanations for this observation exist: the presence of TRAE may be the systemic effect of more active CD8+ T cells which also confer greater antitumor activity; on the other hand, TRAEs may not occur immediately after the first dose, and patients with longer survival had more time for TRAEs to manifest, resulting in an observed increase in TRAE incidence.

There are several limitations to this analysis, including small sample size, single-center nature, heterogeneous population, the inherent biases in a retrospective study, and lack of central blinded independent review for treatment responses. This limits the study to be hypothesisgenerating only and cannot directly recommend the routine clinical use of ipilimumab and nivolumab/ pembrolizumab. Additionally, the subgroup analyses contained small numbers of patients and thus larger-scale studies should be conducted to validate their findings. The OS might have been affected by other subsequent treatments. Finally, most patients were not pretreated with tyrosine kinase inhibitors such as sorafenib before receiving any anti-PD-1 ICI as recommended by current guidelines.

In conclusion, this study demonstrates that using ipilimumab and nivolumab/pembrolizumab as salvage therapy in patients with advanced HCC after tumor progression on prior anti-PD-1/L1 can achieve durable antitumor activity and encouraging survival outcomes with acceptable toxicity. Prospective clinical trials are urgently needed to validate the findings of this hypothesis-generating study and to address the unmet medical need.

Acknowledgements The authors thank the patients and their families, investigators and research staff at Queen Mary Hospital, Hong Kong. The authors also thank the Department of Medicine, Queen Mary Hospital, The University of Hong Kong, for funding support.

Contributors JSLW and TY conceived and designed the study. JSLW, GGWK, VT, BCWL, RL, JC, KWM, WHS, JT, CML, TTC and TY recruited patients and collected the data. JSLW, VT and TY analyzed the data. All authors interpreted the data and were involved in development, review, and approval of the manuscript.

Funding This study was funded by the Research Grant Council-Theme Based Research Fund (T12-704/16-R).

Competing interests TY reports receiving honoraria from Bristol-Myers Squibb and MSD. TY has a consulting or advisory role at Bristol-Myers Squibb and MSD. JC reports receiving honoraria from Bristol-Myers Squibb and has a consulting or advisory role at Bristol-Myers Squibb. JSLW, GGWK, VT, BCWL, RL, KWM, WHS, JT, $\mathrm{CML}$, and TTC have nothing to disclose.

Patient consent for publication Not required.

Ethics approval This study was approved by The University of Hong Kong/Hospital Authority Hong Kong West Cluster Institutional Review Board.

Provenance and peer review Not commissioned; externally peer reviewed.

Data availability statement All data relevant to the study are included in the article or uploaded as supplementary information. The study only utilized deidentified patient data collected from the authors' own institution.

Supplemental material This content has been supplied by the author(s). It has not been vetted by BMJ Publishing Group Limited (BMJ) and may not have been peer-reviewed. Any opinions or recommendations discussed are solely those of the author(s) and are not endorsed by BMJ. BMJ disclaims all liability and responsibility arising from any reliance placed on the content. Where the content includes any translated material, BMJ does not warrant the accuracy and reliability of the translations (including but not limited to local regulations, clinical guidelines, terminology, drug names and drug dosages), and is not responsible for any error and/or omissions arising from translation and adaptation or otherwise.

Open access This is an open access article distributed in accordance with the Creative Commons Attribution Non Commercial (CC BY-NC 4.0) license, which 
permits others to distribute, remix, adapt, build upon this work non-commercially, and license their derivative works on different terms, provided the original work is properly cited, appropriate credit is given, any changes made indicated, and the use is non-commercial. See http://creativecommons.org/licenses/by-nc/4.0/.

\section{ORCID iD}

Jeffrey Sum Lung Wong http://orcid.org/0000-0002-2356-0831

\section{REFERENCES}

1 Bray F, Ferlay J, Soerjomataram I, et al. Global cancer statistics 2018: GLOBOCAN estimates of incidence and mortality worldwide for 36 cancers in 185 countries. CA Cancer J Clin 2018;68:394-424.

2 El-Khoueiry AB, Sangro B, Yau T, et al. Nivolumab in patients with advanced hepatocellular carcinoma (CheckMate 040): an open-label, non-comparative, phase 1/2 dose escalation and expansion trial. Lancet 2017;389:2492-502.

3 Yau T, Park JW, Finn RS, et al. CheckMate 459: a randomized, multi-center phase III study of nivolumab (NIVO) vs sorafenib (SOR) as first-line (1L) treatment in patients (PTS) with advanced hepatocellular carcinoma (aHCC). Ann Oncol 2019;30:v874-5.

4 Zhu AX, Finn RS, Edeline J, et al. Pembrolizumab in patients with advanced hepatocellular carcinoma previously treated with sorafenib (KEYNOTE-224): a non-randomised, open-label phase 2 trial. Lancet Oncol 2018;19:940-52.

5 Finn RS, Ryoo B-Y, Merle P, et al. Pembrolizumab as second-line therapy in patients with advanced hepatocellular carcinoma in KEYNOTE-240: a randomized, double-blind, phase III trial. J Clin Oncol 2020;38:193-202.

6 Finn RS, Qin S, Ikeda M, et al. Atezolizumab plus bevacizumab in unresectable hepatocellular carcinoma. $N$ Engl J Med 2020;382:1894-905.

7 Yau T, Kang Y-K, Kim T-Y, et al. Efficacy and safety of nivolumab plus ipilimumab in patients with advanced hepatocellular carcinoma previously treated with sorafenib: the CheckMate 040 randomized clinical trial. JAMA Oncol 2020. doi:10.1001/jamaoncol.2020.4564. [Epub ahead of print: 01 Oct 2020].

8 Kelley RK, Sangro B, Harris WP, et al. Efficacy, tolerability, and biologic activity of a novel regimen of tremelimumab $(T)$ in combination with durvalumab (D) for patients (PTS) with advanced hepatocellular carcinoma (aHCC). J Clin Oncol 2020;38:4508-08.

9 Zimmer L, Apuri S, Eroglu Z, et al. Ipilimumab alone or in combination with nivolumab after progression on anti-PD-1 therapy in advanced melanoma. Eur J Cancer 2017;75:47-55.

10 Gul A, Stewart TF, Mantia CM, et al. Salvage ipilimumab and nivolumab in patients with metastatic renal cell carcinoma after prior immune checkpoint inhibitors. J Clin Oncol 2020;38:JC01903315.

11 Sternschuss M, Peled N, Allen AM, et al. Can ipilimumab restore immune response in advanced NSCLC after progression on antiPD-1/PD-L1 agents? Thorac Cancer 2020;11:2331-2334.

12 Grimm M-O, Schmidinger M, Duran Martinez I, et al. Tailored immunotherapy approach with nivolumab in advanced renal cell carcinoma (TITAN-RCC). Annals of Oncology 2019;30:v892.

13 Eisenhauer EA, Therasse P, Bogaerts J, et al. New response evaluation criteria in solid tumours: revised RECIST guideline (version 1.1). Eur J Cancer 2009;45:228-47.

14 Seymour L, Bogaerts J, Perrone A, et al. iRECIST: guidelines for response criteria for use in trials testing immunotherapeutics. Lancet Oncol 2017;18:e143-52.

15 National Cancer Institute (US). Common terminology criteria for adverse events (CTCAE). Bethesda, MD US Department of Health and Human Services, National Institutes of Health, National Cancer Institute; 2009.

16 Phase II sequential treatment trial of single agent nivolumab, then combination ipilimumab + nivolumab in metastatic or unresectable Non-Clear cell renal cell carcinoma (ANZUP1602).

17 Study of front line therapy with nivolumab and salvage nivolumab + ipilimumab in patients with advanced renal cell carcinoma.
18 Study of optimized management of nivolumab based on response in patients with advanced RCC (OMNIVORE study).

19 A Clinical Study Evaluating Nivolumab-containing Treatments in Patients With Advanced Non-small Cell Lung Cancer After Failing Previous PD-1/(L)1 Therapy and Chemotherapy.

20 Ipilimumab and nivolumab in patients with Anti-PD-1-axis therapyresistant advanced non-small cell lung cancer.

21 Pembrolizumab and ipilimumab after prior immunotherapy for melanoma.

22 Melero I, Berman DM, Aznar MA, et al. Evolving synergistic combinations of targeted immunotherapies to combat cancer. Nat Rev Cancer 2015;15:457-72.

23 Das R, Verma R, Sznol M, et al. Combination therapy with antiCTLA-4 and anti-PD-1 leads to distinct immunologic changes in vivo. $\mathrm{J}$ Immunol 2015;194:950-9.

24 Gabrilovich DI, Ostrand-Rosenberg S, Bronte V. Coordinated regulation of myeloid cells by tumours. Nat Rev Immunol 2012;12:253-68.

25 Bettelli E, Carrier Y, Gao W, et al. Reciprocal developmental pathways for the generation of pathogenic effector $\mathrm{TH} 17$ and regulatory T cells. Nature 2006;441:235-8.

26 Nowicki TS, Hu-Lieskovan S, Ribas A. Mechanisms of resistance to PD-1 and PD-L1 blockade. Cancer J 2018;24:47-53.

27 Curran MA, Montalvo W, Yagita H, et al. PD-1 and CTLA-4 combination blockade expands infiltrating $T$ cells and reduces regulatory $\mathrm{T}$ and myeloid cells within B16 melanoma tumors. Proc Natl Acad Sci U S A 2010;107:4275-80.

28 Koyama S, Akbay EA, Li YY, et al. Adaptive resistance to therapeutic $\mathrm{PD}-1$ blockade is associated with upregulation of alternative immune checkpoints. Nat Commun 2016;7:10501.

29 Pauken KE, Sammons MA, Odorizzi PM, et al. Epigenetic stability of exhausted $\mathrm{T}$ cells limits durability of reinvigoration by PD-1 blockade. Science 2016;354:1160-5.

30 Hernandez-Gea V, Friedman SL. Pathogenesis of liver fibrosis. Annu Rev Pathol 2011;6:425-56.

31 Henderson NC, Arnold TD, Katamura Y, et al. Targeting of $\alpha \mathrm{V}$ integrin identifies a core molecular pathway that regulates fibrosis in several organs. Nat Med 2013;19:1617-24.

32 Kang N, Gores GJ, Shah VH. Hepatic stellate cells: partners in crime for liver metastases? Hepatology 2011;54:707-13.

33 Friedman SL. Hepatic stellate cells: protean, multifunctional, and enigmatic cells of the liver. Physiol Rev 2008;88:125-72.

34 Affo S, Yu L-X, Schwabe RF. The role of cancer-associated fibroblasts and fibrosis in liver cancer. Annu Rev Pathol 2017;12:153-86.

35 Kudo M, Matilla A, Santoro A, et al. Checkmate-040: nivolumab (NIVO) in patients (PTS) with advanced hepatocellular carcinoma (aHCC) and Child-Pugh B (CPB) status. J Clin Oncol 2019;37:327-27.

36 Antonia SJ, López-Martin JA, Bendell J, et al. Nivolumab alone and nivolumab plus ipilimumab in recurrent small-cell lung cancer (CheckMate 032): a multicentre, open-label, phase 1/2 trial. Lancet Oncol 2016;17:883-95.

37 Yau T, Kang Y-K, Kim T-Y, et al. Nivolumab (NIVO) + ipilimumab (IPI) combination therapy in patients (PTS) with advanced hepatocellular carcinoma (aHCC): results from CheckMate 040. J Clin Oncol 2019;37:4012-12.

38 Hellmann MD, Paz-Ares L, Bernabe Caro R, et al. Nivolumab plus ipilimumab in advanced non-small-cell lung cancer. $N$ Engl J Med 2019;381:2020-31.

39 Sznol M, Ferrucci PF, Hogg D, et al. Pooled analysis safety profile of nivolumab and ipilimumab combination therapy in patients with advanced melanoma. J Clin Oncol 2017;35:3815-22.

40 Larkin J, Hodi FS, Wolchok JD. Combined nivolumab and ipilimumab or monotherapy in untreated melanoma. $N$ Engl J Med 2015;373:23-34.

41 Janjigian YY, Bendell J, Calvo E, et al. CheckMate-032 study: efficacy and safety of nivolumab and nivolumab plus ipilimumab in patients with metastatic esophagogastric cancer. J Clin Oncol 2018;36:2836-44. 